\title{
PERFIL DE CAROTENOIDES DA MICROALGA Chlorella vulgaris EM CULTIVO FOTOTROFICO
}

\author{
D. P. WEBER ${ }^{1}$, P. CAETANO ${ }^{1}$, A. S. FERNANDES ${ }^{1}$, E. JACOB-LOPES ${ }^{1}$, L.Q. ZEPKA ${ }^{1}$
}

${ }^{1}$ Universidade Federal de Santa Maria, Departamento de Ciência e Tecnologia em alimentos. E-mail para contato: lqz@pq.cnpq.br

\begin{abstract}
RESUMO - Microalgas têm sido sistemicamente estudadas devido ao potencial biotecnológico que está associado com a produção de biocompostos de interesse industrial, tais como pigmentos. Em face disso, foram determinados o perfil de carotenoides na biomassa microalgal de Chlorella vulgaris. A microalga foi cultivada em fotobiorreatores operando em regime intermitente, alimentadas com 2,0L de meio BG11. O perfil de carotenoides foi determinado por cromatografia líquida de alta eficiência acoplada a detector fotoarranjo de díodos de espectrômetro de massas (HPLC-PDA-MS/MS). Dezoito carotenoides foram identificados na biomassa microalgal, dentre esses destacam-se os majoritários: all-transequinenona (452.63 $\left.\mu \mathrm{g} \cdot \mathrm{g}^{-1}\right)$, all-trans- $\beta$-caroteno $\left(352.29 \mu \mathrm{g} \cdot \mathrm{g}^{-1}\right)$, 9-cis-equinenona $\left(263.20 \mu \mathrm{g} \cdot \mathrm{g}^{-1}\right)$, all-trans-zeaxantina (271.01 $\left.\mu \mathrm{g} . \mathrm{g}^{-1}\right)$, all-trans-mixoxantofila $\left(129.89 \mu \mathrm{g} \cdot \mathrm{g}^{-1}\right)$.
\end{abstract}

\section{INTRODUÇÃ̃}

Microalgas pertencem a uma classe extremamente diversificada de microrganismos fotossintéticos que apresentam uma grande diversidade em sua composição química e, portanto, isso os torna extremamente atraente para a bioprospeção e potencial exploração como fontes comerciais de uma vasta gama de biomoléculas, incluindo carotenoides (Borowitzka, 2013; Rodrigues et al., 2014). Por estas razões, as microalgas têm sido direcionadas como foco dos processos de biorefinaria economicamente viáveis, visto que, todos os componentes gerados a partir da biomassa de microalgas possuem alto valor para a geração de produtos aplicáveis em diversos setores industriais (Herrero et al., 2015; Suganya et al., 2016; Chew et al., 2017).

Além disso, esses microrganismos são capazes de sintetizar biomoléculas com características estruturais únicas comparadas as obtidas por via química. Tais características estão relacionadas com a atividade antioxidante desses compostos naturais em particular, carotenoides acetilênicos, cetocarotenoides e carotenoides glicosilados (Grama et al., 2014; Rodrigues et al., 2015).

Os antioxidantes astaxantina, $\beta$-caroteno e seus derivados hidroxilo, luteína, licopeno, zeaxantina, equinenona e cantaxantina são os principais carotenoides de valor comercial provenientes de microalgas (Rodrigues et al., 2014; Gong \& Bassi, 2016). Consequentemente, a corrente produção industrial de carotenoides naturais usando microalgas envolve, principalmente, duas espécies, Dunaliella salina e Haematococcus pluvialis, que produzem $\beta$ caroteno e astaxantina, respectivamente. Porém, estudos vem sendo elucidados na busca por 
cultivos de microalgas alternativas que possam suplementar a produção de carotenoides (Přibyl et al., 2016).

Dentre as inúmeras espécies estudadas de microalgas a Chlorella vulgaris, que pertence ao filo das Chlorophyta, é uma alga verde com um diâmetro de 2-10 $\mu \mathrm{m}$, amplamente distribuída em água doce, salobra e marinha e caracteriza-se por ter uma facilidade de cultivo, alta produtividade, alto teor de proteínas e outros componentes bioativos como os carotenoides. Por essas razões, apresenta um amplo potencial para uso em bioprocessos (Nurachman et al., 2015).

Desta forma, o objetivo do estudo foi determinar o perfil de carotenoides contidos na microalga Chlorella vulgaris cultivada em fotobiorreator.

\section{MATERIAL E MÉTODOS}

\subsection{Microrganismos e Meios de Cultura}

A microalga utilizada foi a Chlorella vulgaris. As culturas de reserva foram propagadas e mantidas em ágar-ágar solidificado (20 g.L $\left.\mathrm{L}^{-1}\right)$ contendo meio BG11 sintético (Rippka et al., 1979). As condições de incubação utilizadas foram $25{ }^{\circ} \mathrm{C}$ e intensidade luminosa constante 1 klux.

\subsection{Produção de Biomassa de Microalgas}

A produção de biomassa foi realizada em fotobiorreator integrando duas unidades principais de reação: um fotobiorreator de coluna de bolhas acoplado a uma plataforma de iluminação, operando em regime intermitente com 1,5 L de meio BGN (Ripka et al., 1979). As condições experimentais foram: concentração inicial do inoculo de $100 \mathrm{mg} / \mathrm{L}$, temperatura de $25^{\circ} \mathrm{C}$, relação volume de meio circulante entre a plataforma de iluminação e a zona escura (VI/VE) de $20 \%$, introdução de mistura de ar enriquecido com $15 \%$ de $\mathrm{CO}_{2}$ a uma velocidade equivalente a $1 \mathrm{VVM}$ (volume de ar por volume de meio) e intensidade de luz constante de 13000 Lux.

\subsection{Extração dos Carotenoides}

As extrações dos carotenoides foram realizadas de acordo com o Rodrigues et al., (2015). Os carotenoides foram exaustivamente extraídos com acetato de etila e metanol, transferidos em éter de petróleo/éter etílico, e saponificados overnight (16 horas) a temperatura ambiente com hidróxido de potássio (KOH) 10\% em metanol. Os extratos saponificados foram lavados com água destilada para a remoção do álcali, posteriormente foram concentrados em evaporador rotatório $\left(30^{\circ} \mathrm{C}\right)$. Todas as extrações foram realizadas em triplicata.

\subsection{Análise dos Carotenoides em HPLC-PDA-MS}

A identificação dos carotenoides extraídos da biomassa microalgal foi realizada através de Cromatografia Líquida de Alta Eficiência acoplado a espectrômetro de massas HPLC-PDA- 
MS (Shimadzu, Japão), equipado com detector de arranjo de fotodiodos (PDA) (Shimadzu, Japão) e um espectrómetro de massa com um analisador de armadilha de íons e uma fonte de ionização química à pressão atmosférica (APCI) (modelo Esquire 4000, Bruker Daltonics, Bremem, Alemanha) em coluna C30 YMC ( $5 \mu \mathrm{m}, 250$ x 4,6 mm). Fase móvel de um gradiente linear de metanol e metil terc-butil éter (TBME), de 95:5 para 70:30 em $30 \mathrm{~min}$., para 05:95 em $20 \mathrm{~min}$., permanecendo por $5 \mathrm{~min}$. e retornando a 95:5, com fluxo de 0,9 $\mathrm{mL} / \mathrm{min}$ (De Rosso \& Mercadante, 2007).

A identificação dos carotenoides foi baseada nos parâmetros de ordem de eluição na coluna C30, características do espectro no UV-Visível ( $\lambda$ máx, grau de estrutura fina e intensidade do pico cis) e através de comparação com bibliografia. A quantificação foi realizada por padronização externa, por meio de curva de calibração.

\section{RESULTADOS E DISCUSSOES}

Um total de 18 carotenoides diferentes foram separados no extrato da microalga Chlorella vulgaris (Figura 1). Os carotenoides separados foram identificados com base nas informações combinadas obtidas por eluição cromatográfica, co-cromatografia com padrões, características UV/visível e espectros de massa.

Figura 1: Cromatograma de extrato de carotenoides da biomassa Chlorella vulgaris obtido por HPLC-PDA-MS.

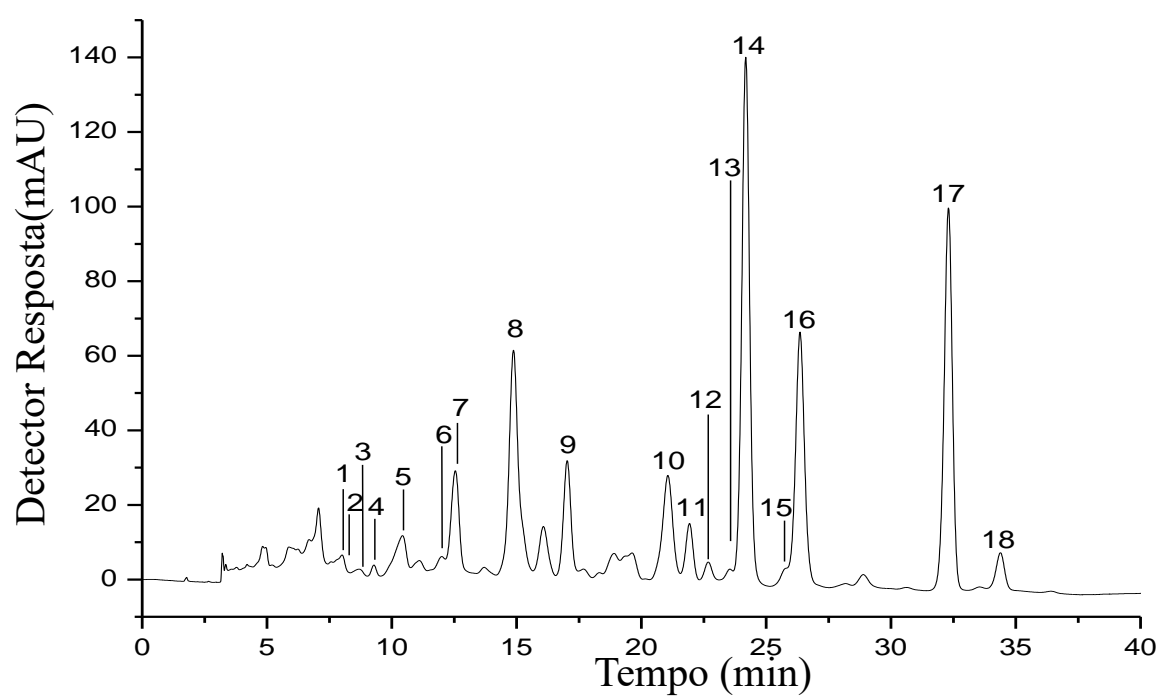

Identificação dos picos: (1) all-trans violaxantina (13.42 $\left.\mu \mathrm{g} \cdot \mathrm{g}^{-1}\right)$; (2) 9-cis-neoxantina (20.53 $\left.\mu \mathrm{g} \cdot \mathrm{g}^{-1}\right)$; (3) all-trans luteoxantina (17.39 $\left.\mu \mathrm{g} . \mathrm{g}^{-1}\right)$; (4) 13-cis-anteroxantina (18.57 $\left.\mu \mathrm{g} . \mathrm{g}^{-1}\right)$; (5) 15-cis-luteína $\left(72.19 \mu \mathrm{g} . \mathrm{g}^{-1}\right)$; (6) all-transdiatoxantina $\left(21.66 \mu \mathrm{g} . \mathrm{g}^{-1}\right)$; (7) all-trans-luteína $\left(111.26 \mu \mathrm{g} \cdot \mathrm{g}^{-1}\right)$; (8) all-trans-zeaxantina $\left(271.01 \mu \mathrm{g} . \mathrm{g}^{-1}\right)$; (9) alltrans-cantaxantina (104.01 $\left.\mu \mathrm{g} . \mathrm{g}^{-1}\right)$; (10) all-trans-mixoxantofila $\left(129.89 \mu \mathrm{g} \cdot \mathrm{g}^{-1}\right)$; (11) $\beta$-carotene-5,6-epoxido (49.23 $\left.\mu \mathrm{g} . \mathrm{g}^{-1}\right)$; (12) all-trans- $\beta$-criptoxantina (15.05 $\left.\mu \mathrm{g} . \mathrm{g}^{-1}\right)$; (13) all-trans-crocoxantina $\left(8.09 \mu \mathrm{g} . \mathrm{g}^{-1}\right)$; (14) alltrans-equinenona (452.63 $\left.\mu \mathrm{g} . \mathrm{g}^{-1}\right) ;(15) 13$ - $\beta$-caroteno $\left(17.17 \mu \mathrm{g} . \mathrm{g}^{-1}\right)$; (16) 9-cis-equinenona $\left(263.20 \mu \mathrm{g} . \mathrm{g}^{-1}\right)$; (17) all-trans- $\beta$-caroteno $\left(352.29 \mu \mathrm{g} . \mathrm{g}^{-1}\right) ;(18) 9$-cis- $\beta$-caroteno $\left(40.43 \mu \mathrm{g} . \mathrm{g}^{-1}\right)$.

Considerando o perfil quantitativo, o extrato mostrou um total de carotenoides de 1977.02 $\mu \mathrm{g} \cdot \mathrm{g}^{-1}$, sendo all-trans-equinenona (pico 14 ) quantitativamente dominante no extrato seguido 
de all-trans- $\beta$-caroteno (pico 17), 9-cis-equinenona (pico 16), all-trans-zeaxantina (pico 8), alltrans-mixoxantofila (pico 10) com teor de carotenoides $452.63 \mu \mathrm{g} . \mathrm{g}-1,352.29 \mu \mathrm{g} . \mathrm{g}-1,263.20$ $\mu \mathrm{g} . \mathrm{g}-1,271.01 \mu \mathrm{g} . \mathrm{g}-1,129.89 \mu \mathrm{g} . \mathrm{g}-1$, respectivamente. Esse valor apresenta um teor substancial quando comparado aos carotenoides obtidos a partir da microalga Phormidium autumnale que apresentou um teor total de carotenoides de 714,3 $\mu \mathrm{g} \cdot \mathrm{g}^{-1}$ (Rodrigues et al., 2015).

Adicionalmente, entre todos os microrganismos capazes de sintetizar carotenoides, as microalgas são melhores produtores de certos materiais bioativos, porque têm um ciclo de vida e uma morfologia mais simples, levando a uma maior produtividade fotossintética (Johnson \& Alric, 2013; Jeon et al.,2016).

\section{CONCLUSÃO}

A biomassa Chlorella vulgaris apresentou a capacidade de produzir um conteúdo significativo de carotenoides, sob condições fotoautotróficas, indicando o potencial como fonte desse pigmento, além de ser uma produção de baixo custo para obtenção de carotenoides. Demonstrando assim, ser consequentemente, uma alternativa viável para aplicação no mercado industrial.

\section{REFERÊNCIAS}

BOROWITZKA, M. A. High-value products from microalgae-their development and commercialisation. Journal of Applied Phycology, v. 25(3), p. 743-756, 2013.

CHEW, K. W.; YAP, J. Y.; SHOW, P. L.; SUAN, N. H.; JUAN, J. C.; LING, T. C.; ... \& CHANG, J. S. Microalgae biorefinery: high value products perspectives. Bioresource Technology, v. 229, p. 53-62, 2017.

DERNER, R. B.; OHSE, S.; VILLELA, M.; CARVALHO, S. M.; FETT, R. Microalgas, produtos e aplicações. Ciência Rural, v. 36, p. 1959-1967, 2006.

GONG, M., \& BASSI, A. Carotenoids from microalgae: A review of recent developments. Biotechnology Advances, v. 34(8), p. 1396-1412, 2016.

GRAMA, B. S.; CHADER, S.; KHELIFI, D.; STENUIT, B.; JEFFRYES, C., \& AGATHOS, S. N. Characterization of fatty acid and carotenoid production in an Acutodesmus microalga isolated from the Algerian Sahara. Biomass and Bioenergy, v. 69, p. 265-275, 2014.

HERRERO, M.; DEL PILAR SÁNCHEZ-CAMARGO, A.; CIFUENTES, A., \& IBÁÑEZ, E. Plants, seaweeds, microalgae and food by-products as natural sources of functional ingredients obtained using pressurized liquid extraction and supercritical fluid extraction. Trends in Analytical Chemistry, v. 71, p. 26-38, 2015.

JOHNSON X, ALRIC J. Central carbon metabolism and electron transport in Chlamydomonas reinhardtii: metabolic constraints for carbon partitioning between oil and starch. Eukaryot Cell, v. 12, p. 776-793, 2013. 
KLASSEN, J. L., \& FOGHT, J. M. Characterization of Hymenobacter isolates from Victoria Upper Glacier, Antarctica reveals five new species and substantial non-vertical evolution within this genus. Extremophiles, v. 15(1), p. 45-57, 2011.

NURACHMAN, Z.; HARTINI, H.; RAHMANIYAH, W. R.; KURNIA, D.; HIDAYAT, R.; PRIJAMBOEDI, B.; ... \& NURBAITI, S. Tropical marine Chlorella sp. PP1 as a source of photosynthetic pigments for dye-sensitized solar cells. Algal Research, v. 10, p. 25-32, 2015.

RIPPKA, R. et al. Generic assignments strain histories and properties of purê cultures of cyanobacteria. Journal General Microbiololy, v. 111, p.1-61, 1979.

RODRIGUES, D. B.; FLORES, É. M.; BARIN, J. S.; MERCADANTE, A. Z.; JACOBLOPES, E., \& ZEPKA, L. Q. Production of carotenoids from microalgae cultivated using agroindustrial wastes. Food Research International, v. 65, p. 144-148, 2014.

RODRIGUES, D. B.; MENEZES, C. R.; MERCADANTE, A. Z.; JACOB-LOPES, E., \& ZEPKA, L. Q. Bioactive pigments from microalgae Phormidium autumnale. Food Research International, v. 77, p. 273-279, 2015.

JEON, S.; JEONG, B. R., \& CHANG, Y. K. Chemicals and Fuels from Microalgae. Consequences of Microbial Interactions with Hydrocarbons, Oils, and Lipids: Production of Fuels and Chemicals, p. 1-21, 2016.

PŘIBYL, P.; PILNÝ, J.; CEPÁK, V., \& KAŠTÁNEK, P. The role of light and nitrogen in growth and carotenoid accumulation in Scenedesmus sp. Algal Research, v. 16, p. 69-75, 2016.

DE ROSSO, V. V., \& MERCADANTE, A. Z. Identification and quantification of carotenoids, by HPLC-PDA-MS/MS, from Amazonian fruits. Journal of agricultural and food chemistry, v. 55(13), p. 5062-5072, 2007.

SUGANYA, T.; VARMAN, M.; MASJUKI, H. H., \& RENGANATHAN, S. Macroalgae and microalgae as a potential source for commercial applications along with biofuels production: A biorefinery approach. Renewable and Sustainable Energy Reviews, v. 55, p. 909-941, 2016. 